\title{
The receptor-binding domain: aptamers (SARS-CoV-2) binding investigation by isothermal titration calorimetry
}

\author{
Vicente Dominguez Arca \\ University of Santiago de Compostela, Spain
}

The aim of the research. To optimize the calorimetry method, which allows to study the binding of the receptor-binding domain (RBD) protein of the coronavirus COVID-19 to aptamer 27 obtained using a computational method.

Material and methods. Computational modeling and calorimetry methods were used to optimize the method.

Results. The results showed that the calorimetry method makes it possible to assess the binding of the RBD protein to the aptamer.

Conclusion. These experiments will be run as soon as will be possible to determine the optimal conditions to functionalize gold substrates with the previously thiolated aptamers.

Key words: aptamer, isothermal titration calorimetry, Spike protein RBD, SARS-CoV-2.

Conflict of interest. The authors declare the absence of obvious and potential conflicts of interest associated with the publication of this article.

Citation: Vicente Dominguez Arca. The receptor-binding domain: aptamers (SARS-CoV-2) binding investigation by isothermal titration calorimetry. Siberian Medical Review. 2021; (2):74-75. DOI: 10.20333/2500136-2021-2-74-75

\section{Procedure fundamentals, proof-of-concept and preliminary results}

The microcalorimetric analyzers are widely used to check the molecular mechanisms of self-assembling, binding or conformational changes. Classically, the isothermal titration calorimetry (ITC) measurements are carried out to obtain the affinity information between proteins and ligands. The fundamentals of the ITC are simple, while its practical implementation is expensive and challenging. Scheme (Fig. 1) try to simplify the mechanisms and critical parts in an ITC apparatus. The kernel of that is the chamber to control the isothermal bath. Inside this chamber, two cells are placed and thermally connected. Reference cell (D) is filled with water or some solvent which thermal capacity response is similar that the solvent employed in the titration experiment. Sample cell (B) is filled with the selected substrate's solution (i.e. protein solution); the key is to observe the system from the surfaces

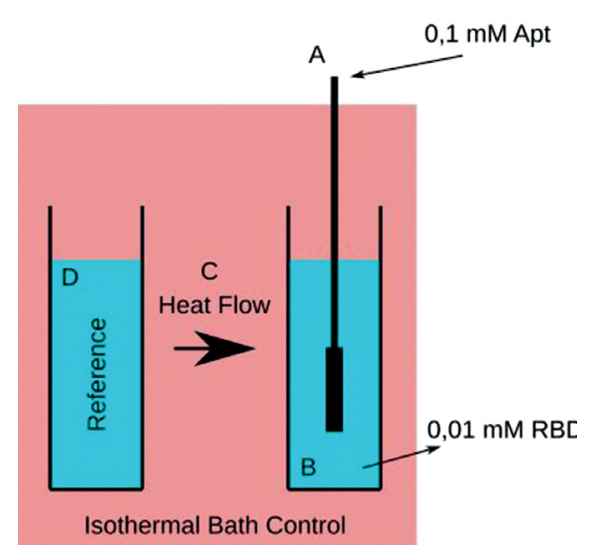

Figure 1. Scheme of the ITC fundamentals. A) syringe to titulation process. B) Sample cell with substrate solution. C) Thermocouple sensor measurement of heat flow. D) Reference cell. physics point of view. In the sample cell always introduce whatever plays the role of substrate, in the syringe (A) we placed the substance that plays the ligand's role. A thermocouple sensor connects the reference and sample cells to record the inter-cells' heat flow. The heat informs about the thermodynamic behaviour at temperature and pressure constant. There are many components involved in this heat: dilution energy of the ligand, interaction energy between substrate and ligand or dilution energy of the formed complexes. The challenges in ITC measurements lie in the calorimetric curves' deconvolution process. The heat flow is contributed for entropic, van der Waals, Coulomb and hydrogen bonding interactions.

In a general sense, during an isothermal and isobaric thermodynamic process, the Gibbs free energy can be written as:

$$
\Delta \mathrm{G}=\Delta \mathrm{H}-\mathrm{T} \Delta \mathrm{S}(1)
$$

Thinking about the equilibrium point is reached by complexes formation saturated, the Gibbs free energy can be written related to the activity of the complexation reaction by the binding constant:

$$
\Delta \mathrm{G}=\mathrm{kBT} \log (\mathrm{K})(2)
$$

A classical ITC experiment is based on one experience of the titration of the ligand on the substrate and another experiment of the titration of the ligand in a solvent-free the substrate. Comparing the calorimetric data can estimate an enthalpy binding value by subtracting both curves and the $\mathrm{K}$ constant by an adequate binding model. The equation (1) allow us to obtain entropic information due to the $\Delta \mathrm{G}$ can be estimated from equation (2).

Our ITC experiences are based on studying the interaction between some aptamers and the receptor-binding domain (RBD) of the Spike protein of SARS-CoV-2. The use of ITC to observe the binding between aptamers and proteins has been recently reported as a powerful tool to obtain relevant information [1-3]. The first step implies to 


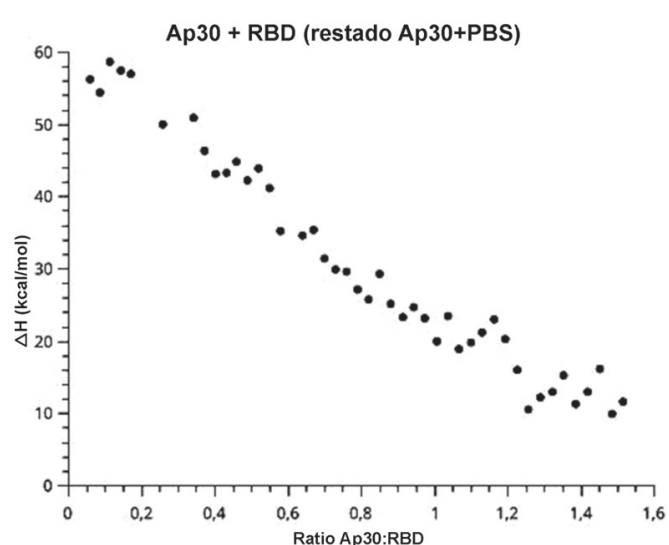

Figure 2. Binding enthalpies from reaction of Apt 30'mer and RBD. The blank information of Apt 30'mer in PBS has been substracted.

determine the binding between selected aptamers and the $\mathrm{RBD}$, once a time this fact will be implemented, the thiolation of the aptamers allows us to incorporate them to gold substrates, and this system could suppose a diagnostic platform to detect variations in the surface plasmon resonance of gold substrates. The selected aptamers $27^{\prime} \mathrm{mer}$ and $30 \mathrm{mer}$ were used to perform several ITC measurements at $35^{\circ} \mathrm{C}$, using that as a ligand to RBD. Both aptamers and RBD were dissolved on PBS at pH 7, 4. In the case of apt 30'mer, two measurements were carried out: one recording data from titration of 30'mer in PBS, and another recording data from titration of 30 'mer in RDB solution. Figure (Fig. 2) shows the enthalpies referent to the binding between 30 'mer and $\mathrm{RBD}$, to plot this graph, the enthalpies from 30'mer in PBS were substrated to enthalpies from $30^{\prime}$ mer in RBD.

The raw data from ITC for 30'mer titrated on RBD were computed with Affinimeter to estimate a preliminary value of $K$. The report of treatment could be revised on supplementary material. The result gives us a value of $\mathrm{Kd}=(7.9666 \pm 0.5691) \mathrm{e}-06 \mathrm{M}-1$, where $\mathrm{Kd}=1 / \mathrm{K}$ of the equation (2). This affinity could be noted as high, though the future experiments will need some modifications in the aptamer's solution to improve the affinity. This ITC experiments' conditions were $5 \mu \mathrm{L}$ per titration during 25 titrations. The goal was to expect the saturation around 12 titrations. The stoichiometry in the equilibrium is $1: 1$ (RBD:Apt), so the concentration of RBD in sample cell was $0.01 \mathrm{mM}$, and due to that the volume of the sample cell was $1.436 \mathrm{~mL}$, we need a syringe concentration of $0.1 \mathrm{mM}$ of the aptamer to observe the saturation around 12 titration. The experiment runs under the isothermal conditions of $35^{\circ} \mathrm{C}$.

Figure (Fig. 3) shows the analogous experiment's enthalpies with 27 'mer aptamer. In this case, the facilities did not allow us to record the contribution of the 27 'mer in PBS; this is a pending task. The volume per titration was reduced to $2.5 \mu \mathrm{L}$, so the total titrations were 50 , and we expect the equilibrium around titration 25 . The experiment runs under the isothermal condition of $35^{\circ} \mathrm{C}$. The raw data from ITC for $30^{\prime}$ mer titrated on RBD were

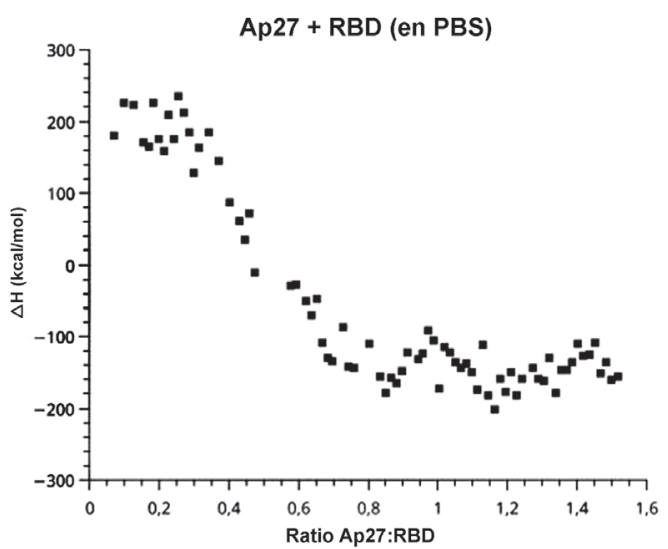

Figure 3. Binding enthalpies from reaction of Apt 27 'mer and RBD. The blank infor-mation of Apt 27 'mer in PBS has not been substracted.

computed with Affinimeter to estimate a preliminary value of $\mathrm{K}$. The report of treatment could be revised on supplementary material. The result gives us a value of $\mathrm{Kd}=(3.0369 \pm 0.4264) \mathrm{e}-07 \mathrm{M}-1$.

Future measurements. On going work

These preliminary results suggest that the 27 'mer aptamer has a higher affinity for RBD than 30 'mer aptamer. More experiments will be carried out to confirm this affinity. Several titrations experiments at different temperatures allow us to explain more in detail the possible enthalpy-entropy compensations between (see eq. 1). On the other hand, a protocol will maximize the aptamers' affinity for RBD; bringing under an increase the temperature until $90{ }^{\circ} \mathrm{C}$ the solution of the aptamer's solution to be continuing with the ITC measurement the affinity should be improved. These experiments will be run as soon as will be possible to determine the optimal conditions to functionalize gold substrates with the previously thiolated aptamers.

\section{References}

1. Amano R, Furukawa T, Sakamoto T. In, Ennifar, E. Microcalorimetry of Biological Molecules: Methods and Protocols, ID: Amano2019; Springer New York: New York; 2019. 268 p.

2. Sakamoto T, Ennifar E, Nakamura Y. Thermodynamic study of aptamers binding to their target proteins. Biochimie. 2018; (145):91-97.

3. Slavkovic S, Zhu Y, Churcher ZR, Shoara AA, Johnson $\mathrm{AE}$, Johnson PE. Thermodynamic analysis of cooperative ligand binding by the ATP-binding DNA aptamer indicates a population-shift binding mechanism. Scientific Reports. 2020;(10):18944.

\section{Author information}

Vicente Dominguez Arca, PhD student, University of Santiago de Compostela; Address: Colexio de San Xerome, Praza do Obradoiro, s/n., 15782, Santiago de Compostela, A Coruña, Spain; Phone: +34(605)474040; e-mail: vicente.dominguez@rai.usc.es

Received 17 February 2021 Revision Received 18 March 2021 Accepted 31 March 2021 